$10 \mathrm{~Hz}$ and the amplitude set at equal to the MAP value at the beginning, was increased, if necessary, until the infant's chest was seen to be "bouncing". In the HFOV+VG mode, the VThf was set at $2 \mathrm{ml} / \mathrm{kg}$ initially on the basis of our clinical experience. The Amplitude limit was set at $15-20 \%$ above the average amplitude needed to achieve the target VTHf. Moreover during each $2 \mathrm{~h}$ observation period, the following variables were continuously display at 5-min intervals: FiO2, MAP, VThf, Carbon dioxide diffusion co efficiency (DCO2), Amplitude (DeltaPhf), from the ventilator records and heart rate, mean blood pressure, $\mathrm{SpO} 2$ from the standard cardiorespiratory monitor.

Results The mean gestational age was 28,2 (24-32) week and the mean gestational weight was 1087 (704-1960) gr. There was no significant difference in the mean PCO2, FiO2, DeltaPhf, MAP, VTHf, DCO2, Minute ventilation (MVe), Dynamic compliance (CDyn), Resistance (R). Hypocarbia event (PCO2 <40 $\mathrm{mmHg}$ ) occurred eleven (\%36) sample during $\mathrm{HFOV}+\mathrm{VG}$ period against seven sample (\%23) during HFOV period but not statistical significant.

Conclusion This preliminary result demonstrated that VG option, when combined with HFOV, a stable and feasible ventilation mode for neonatal patients and can achieve equivalent gas exchange After a careful analysis of the results, a set VTHf of $1,5 \mathrm{ml} / \mathrm{kg}$ seems to be successful achieving equivalent gas exchange using lover airway pressure.

\section{PO-0742 HYPOTHERMIA A RISK FACTOR FOR RESPIRATORY DISTRESS SYNDROME IN PREMATURE INFANTS?}

${ }^{1} \mathrm{C}$ Jensen, ${ }^{2} \mathrm{~F}$ Ebbesen, ${ }^{3} \mathrm{JP}$ Petersen, ${ }^{3} \mathrm{AS}$ Sørensen, ${ }^{3} \mathrm{~TB}$ Henriksen. ${ }^{1}$ Department $A f$ Pediatrics, Aarhus University Hospital, Aarhus N, Denmark; ${ }^{2}$ Department Af Pediatrics, Aalborg University Hospital, Aalborg, Denmark; ${ }^{3}$ Department Af Pediatrics, Aarhus University Hospital, Aarhus, Denmark

\subsection{6/archdischild-2014-307384.1381}

Background Hypothermia isgenerally thought to be a risk factor of respiratory distress syndrome (RDS) in premature infants. However, previous studies have primarilyinvestigated the association between hypothermia and death.

Aim To investigate the association between body temperature and severe RDS.

Methods The study population consists of all infants born before 32 weeks of gestationand admitted to the neonatal intensive care unit (NICU), Aalborg UniversityHospital, Denmark April 1997 and December 2011. Rectal temperature was measuredat admission. Severe RDS was defined as the need for surfactant treatment or death within the first 3 days of life in premature infants bornbefore 32 weeks gestation. Data are provided bynational registries and will be analysed by logistic regression while adjusting formarkers of infection, gestational age, time from delivery to admission, asphyxiaand a proxy variable for fetal growth restriction.

Results Preliminary results from 593 infants show that64\% (n = 381) had hypothermia $\left(<36.5^{\circ} \mathrm{C}\right), 33 \%(\mathrm{n}=197)$ had arectal temperature within the normal range $\left(36.5^{\circ} \mathrm{C}-37.5^{\circ} \mathrm{C}\right)$ and $3 \%$ $(\mathrm{n}=15)$ had hyperthermia $\left(>37.5^{\circ} \mathrm{C}\right)$. The unadjusted odds for need for surfactantif hypothermic were almost twice the odds in normothermic newborns at admission (OR 1.92 95\% CI: 1.34; 2.76). Further analyses are ongoing and refined resultswill be presented.

Conclusions In very preterm neonates the unadjusted odds of severe RDS was almosttwo times higher if they had hypothermia at admission compared to those withnormotermia.

\section{PO-0743 USE OF A NEW-GENERATION ELECTRONIC MICROPUMP NEBULISER TO DELIVER BUDESONIDE IN CHRONIC LUNG DISEASE: A FEASIBLE ALTERNATIVE TO SYSTEMIC DEXAMETHASONE?}

${ }^{1} \mathrm{~S} J o b,{ }^{2} \mathrm{~A}$ Kopuri, ${ }^{2} \mathrm{~K}$ Ives, ${ }^{1} \mathrm{P}$ Clarke. ${ }^{1}$ Neonatal Unit, Norfolk and Norwich University Hospitals NHS Foundation Trust, Norwich, UK; ${ }^{2}$ Neonatal Unit, John Radcliffe Hospital Oxford University NHS Trust, Oxford, UK

\subsection{6/archdischild-2014-307384.1382}

Background and aim Inhaled corticosteroids reduce lung inflammation in chronic lung disease (CLD) and may be safer than systemic dexamethasone treatment, but evidence of better efficacy is lacking. State-of-the-art aerosol delivery systems may permit enhanced alveolar steroid delivery compared with traditional metered-dose inhalers/spacers or jet nebulisers. We evaluated a new-generation electronic micropump vibrating-mesh nebuliser for topical airways delivery of budesonide in infants with severe CLD requiring nasal high-flow respiratory support.

Methods We reviewed our units' clinical experience of delivering budesonide via the Vapotherm ventilation circuit to infants with established CLD using the Aeroneb Pro-X (Aerogen, Ireland) nebuliser.

Results 7 babies with severe CLD received nebulised budesonide since 2013. Median (range) birth gestational age was 26.9 (23.1-27.7) weeks, birthweight 720 (490-850) g. Nebulisation commenced at age 62 (29-104) days postnatal, by which time 6 babies had accumulated 33 (10-49) days' systemic dexamethasone. Initial budesonide dosage was $0.5 \mathrm{mg} /$ dose administered 2 4 times/day. Duration of nebulisation prior to discharge/back transfer was 55 (9-69) days. Nebulisation permitted successful weaning from dexamethasone within 8 (0-20) days in 6 babies and obviated the need for systemic dexamethasone in another. After starting nebulisation, no baby needed a subsequent oral dexamethasone course before discharge/back transfer.

Conclusion Use of a new-generation electronic micropump nebuliser for topical airways budesonide delivery to nasal high-flow dependent infants is feasible and may avoid the need for systemic dexamethasone. The comparative safety and efficacy of this new technology for steroid delivery to ventilatory supportdependent CLD babies should now be formally examined in clinical trials.

\section{PO-0744 GENOME-WIDE ASSOCIATION STUDY OF BRONCHOPULMONARY DYSPLASIA}

${ }^{1}$ MK Karjalainen, ${ }^{1} \mathrm{M}$ Mahlman, ${ }^{1} \mathrm{JM}$ Huusko, ${ }^{2} \mathrm{~S}$ Andersson, ${ }^{2} \mathrm{~A}$ Kari, ${ }^{3} \mathrm{~L}$ Lehtonen, ${ }^{4} \mathrm{U}$ Sankilampi, ${ }^{5} \mathrm{O}$ Tammela, ${ }^{1} \mathrm{R}$ Marttila, ${ }^{1} \mathrm{M}$ Rämet, ${ }^{1} \mathrm{M}$ Hallman. ${ }^{1}$ Department of Pediatrics, University of Oulu and Oulu University Hospital, Oulu, Finland; ' ${ }^{2}$ Children'S Hospital, Helsinki University Central Hospital and University of Helsinki, Helsinki, Finland; ${ }^{3}$ Department of Pediatrics, Turku University Hospital, Turku, Finland; ${ }^{4}$ Department of Pediatrics, Kuopio University Hospital, Kuopio, Finland; ${ }^{5}$ Department of Pediatrics, Tampere University Hospital, Tampere, Finland

\subsection{6/archdischild-2014-307384.1383}

Background and aims Bronchopulmonary dysplasia (BPD) is the most common chronic disease associated with very preterm birth. BPD has a significant genetic background but the predisposing genes are insufficiently known. The aim is to find genetic factors that predispose to moderate-severe BPD using a hypothesis-free, genome-wide approach.

Methods The study populations included preterm infants (gestational age $<31$ weeks) born during 1997-2013 in Oulu 